\title{
Antibiotic screening of urine culture as a tool for interal quality audit
}

\author{
Sreenivasan Srirangaraj, Arunava Kali, MV Pravin Charles
}

Department of Microbiology, Mahatma Gandhi Medical College \& Research Institute,

Pondicherry

\section{THIS ARTICLE HAS BEEN RETRACTED DUE TO PLAGIARISM.}

\section{RESEARCH}

Please cite this paper as: Srirangaraj S, Kali A, Charles MVP. Antibiotic screening of urine culture as a tool for internal quality audit. AMJ 2014, 7, 2, 73-77. http://doi.org/10.21767/AMJ.2014.1956

\section{Corresponding Author:}

Dr. S. Srirangaraj

Dept. of Microbiology, Mahatma Gandhi

Medical College and Research Institute,

Pondicherry, India.

Email: rangaraj.sreenivasan@gmail.com

\section{Abstract}

\section{Background}

Microbiologic culture of urine is an important component in the diagnosis of urinary tract infections (UTI). However, confounding factors like the patient's current history of antibiotic usage can affect the interpretation of urine culture results.

\section{Aims}

This study was undertaken to find out the occurrence of unreported antibiotic consumption prior to urine culture and its clinical consequences.

\section{Method}

In this descriptive study conducted from February to August 2013, we tested consecutive urine specimens for the presence of antibiotics with a modified urine antibacterial substance assay (UABA) and compared the results obtained with the accuracy of antibiotic data entry on the accompanying request forms. In addition, the consequences of culturing urine specimens with incomplete antibiotic history received in the laboratory were investigated.

Results

Out of 2,040 urine specimens, 721 (35.34 per cent) were
UABA positive. Comparison of antibiotic screening of urine with antibiotic data entry from request forms showed 1,299 (63.68 per cent) true-negative, 38 (1.86 per cent) truepositive, 20 (0.98 per cent) false-negative, and 683 (33.48 per cent) false-positive results. The sensitivity and specificity of this test were 65.52 per cent and 65.54 per cent, respectively. The positive and negative predictive values were 5.27 per cent and 98.48 per cent, respectively. The UABA had a positive diagnostic likelihood ratio of 1.901 .

\section{Conclusion}

This internal audit demonstrates how the accurate history of current antibiotic usage in the request forms impacts the interpretation of urine culture results.

\section{Key Words}

Antibiotic screening; internal audit; Antibiotic History; Urine culture

\section{What this study adds:}

\section{What is known about this subject?}

Easy availability of over the counter antibiotics in India often results in unreported antibiotic use. Antibiotics, when used for urinary tract infections, become an important confounding factor in the correct interpretation of urine culture results.

\section{What new information is offered in this study?}

We found that more than one-third of the urine specimens had antibiotic activity, while documented history of antibiotic usage was available for only 2.84 per cent of the samples.

3. What are the implications for policy, research or practice?

This internal audit practice demonstrates the importance of current history of antibiotic usage in the correct interpretation of urine culture results.

\section{Background}

Urinary tract infections (UTIs) are usually diagnosed on the basis of history, physical examination, urinalysis and urine 


\section{THIS ARTICLE HAS BEEN RETRACTED DUE TO PLAGIARISM.}

culture. Urine samples received in the microbiology laboratory are subjected to semi-quantitative culture to determine whether they contain a potentially pathogenic bacterium in sufficient numbers to identify it as the causal infecting organism (Significant Bacteriuria). ${ }^{1}$

A number of confounding factors affect the interpretation of urine specimens received for culture in the clinical microbiology laboratory. Antibiotic history is often not included by physicians on the laboratory request forms or patients do not admit to taking antibiotics prior to urine culture collection. This information is important when correlating culture results with other laboratory findings. ${ }^{2}$ This study was conducted to demonstrate unreported antibiotic consumption prior to urine culture and its clinical consequences.

\section{Method}

\section{Detection of antibacterial activity in the urine}

We followed the modified urine antibacterial substance assay (UABA) described by Wilson et al., ${ }^{2}$ a technique which was originally described by Sombrero et al. ${ }^{3}$ and others ${ }^{4,5}$ by using two Mueller-Hinton agar plates each inoculated with a lawn of Escherichia coli ATCC 25922 and Staphylococcus aureus ATCC 25923. The ATCC strains were suspended in 0.85 per cent sterile saline and adjusted to a 0.5 McFarland standard using a nephelometer. A sterile cotton swab was dipped into the standardised suspension and excess fluid removed by rotation of the swab against the side of the tube. The plates were then inoculated by streaking the swab all over the surface of the medium (lawn culture) three times, by rotating the plate through an angle of $60^{\circ}$ after each application as is done for the modified Kirby-Bauer method. ${ }^{6}$ Autoclaved sterile discs of $6 \mathrm{~mm}$ diameter were prepared from Whatman Grade 1 filter paper (Whatman International, Ltd., Maidstone, England). After the inoculum dried, the discs were placed aseptically on the inoculated plates at a distance of $10-12 \mathrm{~mm}$ apart. One loopful of urine $(1 \mu l)$ was transferred from each urine specimen on to the correspondingly labelled autoclaved disc aseptically.

Quality control (QC) was ensured by placing one set as a negative control, i.e., discs without antibiotics. For S. aureus ATCC 25923, another set was inoculated with a panel of gram positive standard antibiotic impregnated discs comprising penicillin (10 units), ampicillin (10 $\mu \mathrm{g})$, gentamicin $(10 \mu \mathrm{g})$, norfloxacin $(10 \mu \mathrm{g})$, cefotaxime $(30 \mu \mathrm{g})$ and vancomycin $(30 \mu \mathrm{g})$. Similarly, a panel of gram-negative antibiotic discs comprising nitrofurantoin $(300 \mu \mathrm{g})$, nalidixic acid $(30 \mu \mathrm{g})$, norfloxacin $(10 \mu \mathrm{g})$, cefotaxime $(30 \mu \mathrm{g})$, gentamicin $(10 \mu \mathrm{g})$, and imipenem $(10 \mu \mathrm{g})$ were used for testing E. coli ATCC 25922. QC was passed when the negative control showed no zone of inhibition around the disc and the positive control showed a zone of inhibition of appropriate size around the disc as per CLSI guidelines. ${ }^{7}$

\section{Urine cultures}

All urine specimens received in the laboratory from 1 February 2013 to 31 August 2013 were processed using standard methods for processing urine specimens, ${ }^{1}$ at the Department of Microbiology, Mahatma Gandhi Medical College \& Research Institute, Pondicherry, India. Specimens were inoculated onto Blood agar and Cystine Lactose Electrolyte Deficient (CLED) culture medium within two hours of collection and incubated overnight at $35-37^{\circ} \mathrm{C}$. Any growth on the media, i.e., growth of pathogens, no significant bacteriuria, mixed growth as well as growth of contaminants were considered as a positive urine culture for the purpose of this study. ${ }^{2}$

\section{Modified Urine Antibacterial Substance Assay (UABA)}

Simultaneously each urine sample was inoculated onto an individual disc on each Mueller-Hinton plate (one with Escherichia coli ATCC 25922 and the other Staphylococcus aureus ATCC 25923) using a $1 \mu \mathrm{L}$ loop. Each disc on both plates was labelled with the corresponding urine sample number and incubated overnight at $35-37^{\circ} \mathrm{C}$ along with the routine culture plates. Any zone of inhibition around the disc was considered positive for antibacterial activity. Figure 1 shows the modified UABA (Urine Antibacterial substance Assay) using E.coli ATCC 25922 and S.aureus ATCC 25923.

\section{Figure 1: Modified UABA using E.coli ATCC 25922 and Staph.aureus ATCC 25923}

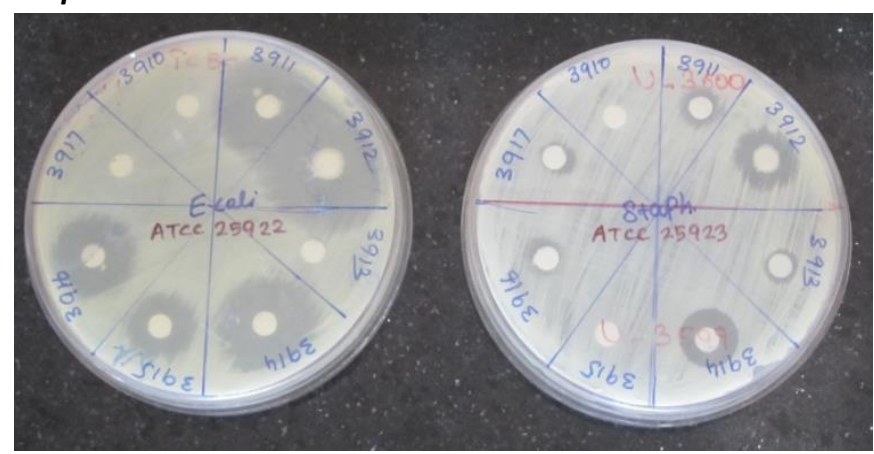

Statistical analyses included descriptive statistics like percentages and proportions. All statistical analyses were performed using SPSS software (version 16.0, SPSS, Chicago, USA).

\section{Results}




\section{THIS ARTICLE HAS BEEN RETRACTED DUE TO PLAGIARISM.}

The results of the urine culture and UABA are depicted in Table 1.

Table 1: Results of urine culture compared with UABA test

\begin{tabular}{|l|l|l|l|}
\hline & UABA + & UABA - & Total \\
\hline Culture + & 288 & 710 & 998 \\
\hline Culture - & 433 & 609 & 1,042 \\
\hline Total & 721 & 1,319 & 2,040 \\
\hline
\end{tabular}

From a total of 2,040 consecutive urine specimens submitted for culture and susceptibility, 998 (48.92 per cent) samples yielded positive bacterial cultures and 721 (35.34 per cent) were UABA positive. Of 721 UABA positive samples, no growth was detected in 433 (60.1 per cent) samples, 243 (33.7 per cent) showed growth of pathogens, while 5 (0.7 per cent) showed insignificant growth, and 40 (5.55 per cent) showed mixed growth or growth of contaminants.

Comparison of antibiotic screening of urine with antibiotic data entry from request forms was done. True-positive was defined as those urine specimens that were UABA positive and antibiotic history $(\mathrm{AH})$ from request forms was available. True-negative was defined as those urine specimens that were UABA negative and antibiotic history was not specified. False-positive was defined as those urine specimens that were UABA positive but antibiotic history was negative. Lastly, false-negative were those urine specimens that were UABA negative but had antibiotic history.

The data obtained from the 2,040 urine samples as depicted in the Table 2, showed 1,299 (63.68 per cent) true negative, 38 (1.86 per cent) true positive, 20 (0.98 per cent) false negative, and 683 (33.48 per cent) false positive results. The sensitivity and specificity of this test was 65.52 per cent and 65.54 per cent, respectively. The positive and negative predictive values were 5.27 per cent and 98.48 per cent, respectively. The UABA had a positive diagnostic likelihood ratio of 1.901 ( $p$ value $<0.0001$, very significant).

Table 2: UABA as a diagnostic tool with Antibiotic History $(\mathrm{AH})$ obtained from request forms

\begin{tabular}{|l|l|l|l|}
\hline & $\mathrm{AH}+$ & $\mathrm{AH}-$ & Total \\
\hline UABA+ & 38 & 683 & 721 \\
\hline UABA - & 20 & 1,299 & 1,319 \\
\hline Total & 58 & 1,982 & 2,040 \\
\hline
\end{tabular}

Of the 721 UABA positive samples, 28 (3.9 per cent) had leukocyte counts $>10 /$ high power field (HPF), 150 (20.8 per cent) of which were received from outpatients and 571 (79.2 per cent) from inpatients. Of these 571 UABA positive samples from inpatients, 339 (59.4 per cent) reported no growth

Lower colony counts of $<10^{3}$ were seen in 130 samples out of 2,040 samples. Of these, 35 (26.9 per cent) were UABA positive and antibiotic history was given in only 7 (5.4 per cent) samples.

Compliance in obtaining antibiotic histories for inpatients (37; 97.37 per cent) was higher than that reported for outpatients ( $1 ; 2.63$ per cent).

\section{Discussion}

The true-positive UABA results correlated well with transcribed history provided on the laboratory request forms and the false-negatives could indicate inert drug compounds, counterfeit anti-infective drugs, and/or the patient's ignorance of the content of medicine consumed. ${ }^{2}$

The false-negative UABA could also indicate the empiric antibiotic to be used by the physician in the treatment of the suspected UTI. In our study, 60.1 per cent of positive UABAs showed no growth, re-emphasising the fact from earlier studies ${ }^{2,8-10}$ that samples sent to the microbiology laboratory during a course of antibiotic treatment may not yield clinically significant results.

In this study, a total of 683 (33.48 per cent) false-positive results were detected by UABA test. The high numbers of false-positive results indicate the use of antibiotics prior to culture and/or other antibacterial substances present in the urine. This could be due to: (1) the history was not taken properly; (2) the patient either could not or was unwilling to give history due to ignorance of content of medicine, ${ }^{3}$ poor recall, or intentional false denial due to self-perceived misuse of drugs; or (3) the antibiotic treatment of a patient with infection other than urinary tract infection. Beers et al. found that medication histories were frequently inaccurate due to failure to obtain or document the antibiotic taken or patient denial of usage. ${ }^{11}$ Inaccurate interpretation of culture reports and promoting the emergence of drug resistance can result from this practice. ${ }^{12}$ Leading questions regarding drug history by using the brand names of antibiotics can reduce the recall errors. ${ }^{3}$

The absence of pyuria provides strong evidence against the presence of urinary tract infection. ${ }^{10,12}$ However, sterile pyuria (3.9 per cent of cases with pus cells $\geq 10 / \mathrm{HPF}$ in our 


\section{THIS ARTICLE HAS BEEN RETRACTED DUE TO PLAGIARISM.}

study) should also induce a high suspicion of prior antibiotic usage. $^{1}$

Another interesting finding in this study was that roughly one-fourth [35 (26.9 per cent) out of 130] samples with low colony counts $\left(<10^{3} \mathrm{CFU} / \mathrm{ml}\right)$ were UABA positive and antibiotic history was given in only 7 (5.4 per cent) samples. This means in the absence of history of prior antibiotic usage, these samples would have wrongly been interpreted as "No Significant Bacteriuria", when in reality they had lower colony counts due to prior antibiotic usage and were to be reported as significant as was done in this study.

Failure to either perform urine cultures or to correctly interpret their results may lead to therapeutic failures. UTI diagnosis can be further complicated by the presence of antibiotics in urine specimens submitted for culture, particularly from areas where they are readily purchased over the counter, without prescriptions..$^{2-4,8}$ This situation can compromise the recovery of bacterial pathogens and their accurate colony count, resulting in false-negative results and diagnostic dilemmas, especially in symptomatic patients. Bacterial counts per $\mathrm{ml}$ urine can be temporarily reduced by antibiotics, causing a transient remission of clinical symptoms, thus confounding the efficacy of treatment in chronic or recurrent asymptomatic infections. $^{13}$

Obtaining recent antibiotic history from a patient and including it in the request forms is often neglected, and this diagnostic information can influence interpretation of culture results. Limited sensitivity of urine cultures due to prior antibiotic consumption enforces the need to take proper history and avoid sending such samples for culture to the microbiology lab. Such periodic laboratory audits could significantly improve patient care, limit misuse of antibiotics, and have a lasting impact on the quality of the laboratory report.

\section{Conclusion}

A periodic internal audit using the UABA described here to measure the presence of antibiotics in urine cultures can prove to be a useful quality assurance tool to determine the impact on urine culture interpretation. This could raise awareness regarding the importance of obtaining antibiotic history using an evidence-based protocol. An internal quality improvement program can include UABA monitoring on a continuous basis, which might trigger a change in physicians' practices and may lead to overall quality improvement in the management of urinary tract infections.

\section{References}

1. Collee JG, Fraser AG, Marmion BP, Simmons A. Mackie \& McCartney Practical Medical Microbiology. $14^{\text {th }}$ ed: Churchill Livingstone; 2010.

2. Wilson G, Badarudeen S, Godwin A. Antibiotic screening of urine culture as a useful quality audit. J Infect Dev Ctries. 2011;5:299-302.

3. Sombrero $L$, Sunico $M E$, Quiambao $B$, Lucero $M$, Gatchalian S, Leinonen $M$, et al. Reliability of parental history of antibiotic use for Filipino children admitted with acute lower respiratory tract infection. Am J Trop Med Hyg. 1999;60:397-9.

4. Abu Shaqra QM. Antimicrobial activity in urine: effect on leukocyte count and bacterial culture results. New Microbiol. 2001;24:137-42.

5. Wallenstein FA, Ringelmann R. Evaluation of successful chemotherapy of infections of the urinary system by determination of antibacterial activity in the urine (author's transI). Immun Infekt. 1976;4:276-8.

6. Vandepitte J, Verhaegen J, Engbaek K, Rohner P, Piot P, Heuck CC. Basic laboratory procedures in clinical bacteriology. 2nd ed. Singapore: World Health Organization; 2003.

7. Clinical and Laboratory Standards Institute. Performance standards for antimicrobial susceptibility testing: 23rd informational supplement. CLSI document M100-S23. Wayne, Pennsylvania: Clinical and Laboratory Standards Institute; 2013.

8. Catalano M, Almiron MA, Romeo AM, Caruso E, Murtagh $P$, Harisiadi J. Comparison between parental report and results of microbiologic agar assay for presence of antibiotic in urine of Argentinian children with acute lower respiratory tract infection. Rev Infect Dis. 1990;12 Suppl 8:S998-1000.

9. Ansorg R, Zappel H, Thomssen R. Significance of the antibacterial agent assay of urine for bacteriological diagnosis and control of chemotherapy of urinary tract infections (author's transl). Zentralbl Bakteriol Orig A. 1975;230:492-507.

10. Endeman $H$, Schelfhout V, Voorn GP, van Velzen-Blad $H$, Grutters JC, Biesma DH. Clinical features predicting failure of pathogen identification in patients with community acquired pneumonia. Scand J Infect Dis. 2008;40:715-20.

11. Beers $M H$, Munekata $M$, Storrie $M$. The accuracy of medication histories in the hospital medical records of elderly persons. J Am Geriatr Soc. 1990;38:1183-7.

12. Hoberman A, Wald ER, Reynolds EA, Penchansky L, Charron $M$. Pyuria and bacteriuria in urine specimens obtained by catheter from young children with fever. J Pediatr. 1994;124:513-9. 
THIS ARTICLE HAS BEEN RETRACTED DUE TO PLAGIARISM.

13. Barsanti JA, Finco DR. Laboratory findings in urinary tract infections. Vet Clin North Am Small Anim Pract. 1980;9:729-48.

\section{ACKNOWLEDGEMENTS}

We express our sincere thanks to Ms. Priya and Ms. Tamizharasi for their dedicated technical assistance towards this audit.

\section{PEER REVIEW}

Not commissioned.

\section{CONFLICTS OF INTEREST}

The authors declare that they have no competing interests. 